\title{
Capabilities of modal type liquid crystal spatial modulators for formation of light fields with orbital angular momentum
}

\author{
Svetlana Kotova*, Aleksandra Mayorova, and Sergey Samagin \\ Lebedev Physical Institute, Samara Branch, 221 Novo-Sadovaya Str., Samara, 443011 Russia
}

\begin{abstract}
The capabilities of modal LC modulator (LC focusator) for optical vortices formation are demonstrated. Two options of changing of the contact electrodes geometry providing a voltage jump and accordingly the jump for profile of phase transmission of modulator are proposed. The examples of distributions of intensity and phase for generated fields are presented.
\end{abstract}

Light fields with complex spatial structure and orbital angular momentum are very promising for contemporary optical technologies. Fields like that are useful for optical diagnostics, information processing, laser diagnostics, optical manipulation etc. [1]. The range of problems that can be solved using complex light fields, is constantly expanding [2]. Thus, the realization and improvement of methods for formation of such fields and for dynamic control of their characteristics is important. At the same time, for practical applications inexpensive, technologically simple devices working in a wide spectral region and characterized by relatively low light loss are of interest. Liquid crystal (LC) light modulators of modal type, and, in particular, LC focusator developed at Samara Branch of Lebedev Physical Institute can be used as such a device. Modal type modulators include in their design a high-resistance layer performing the function of the control electrode. The phase delay profile is generated by changing the voltage distribution applied to the electrodes as well as the reorientation of molecules of LC layer between the glass substrates covered with high-resistance and low-resistance coatings.

The aim of this work is to demonstrate the enhanced functionality of the LC focusator to generate light beams with angular orbital momentum by means of changing the contact electrodes geometry. Previously it was shown that the LC focusator allows one to focus light field into the point spot or line, and also to generate transverse intensity distributions in the form of various contours, while the sizes, positions and orientations can be controlled with the applied voltages [3-5]. But all fields generated with the LC focusator are characterized by zero orbital angular momentum, which limits their application.

To generate beams with orbital angular momentum (optical vortices) it is necessary to ensure the phase jump. Two ways of changing the configuration of the contact electrodes are proposed. In the first case, the contact electrode on one of substrates is divided by half, and this region is extended down to the middle of the aperture. And in the second case, two narrow

Corresponding author: kotova@,fian.smr.ru 
linear electrodes are applied from the centre to the edge in the middle of the free side of one of the substrates. In both cases we have two additional closely spaced electrodes and we can realize a voltage jump and, therefore, the jump in the profile of phase delay applying to those electrodes the selected slightly different in magnitude potentials. The result is generation of angular orbital momentum for the light passing through LC modulator or reflected from it.

The results of simulation showed that in the first case it is possible to implement the phase delay like to the spiral axicon and to form the transverse intensity distribution in the shape of a ring at some distance from the LC modulator. To ensure more homogeneous phase distribution and intensity of the generated fields it is preferable to use the dual-frequency control mode. The control of voltage and phase delay distributions and respectively of the generated fields can be achieved by changing the potentials on one of the frequencies. The intensity and phase distributions of the light field generated by means of the LC modulator with dual-frequency control mode at a distance of $25 \mathrm{~cm}$ from it are presented as example in Fig. 1 (a, b).

The proposed configuration of modal LC focusator with additional narrow electrodes can be used as a spiral plate, which is clearly illustrated by the examples of intensity and phase distributions of the generated light fields in the far zone of diffraction shown in Fig. 1 (c, d). The field was calculated in the focal plane of the lens with a focal length of $1 \mathrm{~m}$ for the device with aperture $2 \times 2 \mathrm{~mm}$, limited by a diaphragm with a diameter of $1 \mathrm{~mm}$.

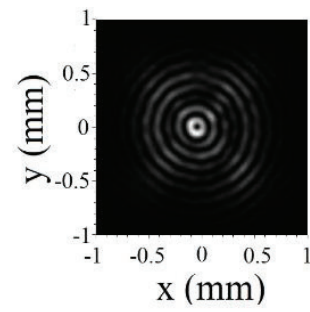

a

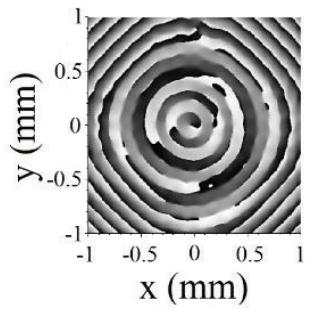

b

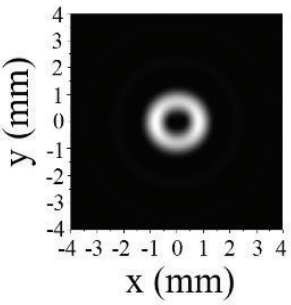

c

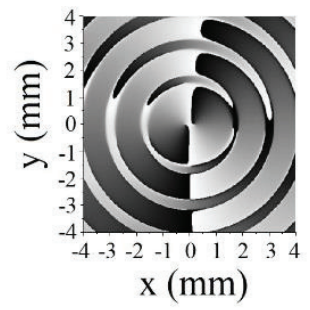

d

Fig. 1. The distributions of intensity $(a, c)$ and phase (b, d) of light fields generated by LC focusator. a, $\mathrm{b}$ - The simulation results for spiral LC modulator with dual-frequency control mode at a distance of $25 \mathrm{~cm}$ from it. c, d - The simulation results for the LC focusator with additional electrodes at the focal plane of the lens.

The LC focusator is a technologically simple and relatively low-cost device. Besides, the use of solid electrodes allows us to form a smooth continuous profile of the phase delay and to control it smoothly. Due to these features in our opinion the suggested configurations of electrodes can be useful for the optical vortexes formation in optical manipulations tasks.

This research is financially supported by the Russian Foundation for Basic Research (projects No. 1602-01055 and No. 16-42-630773).

\section{References}

[1] M. Padgett, Opt. Express 25, 11265 (2017)

[2] H. Rubinsztein-Dunlop et al., J. Opt. 19, 013001 (2017)

[3] A. Korobtsov, S. Kotova, N. Losevsky et al., J. Opt. 16, 035704 (2014)

[4] S. Kotova, A. Mayorova, S. Samagin, J. Opt. 17, 055602 (2015)

[5] S. Kotova, A. Mayorova, S. Samagin, J. Opt. Technol. 84, 323 (2017) 